\title{
Beam Coupling Phenomena in Fast Kicker Systems*
}

\author{
W. Zhang, L. A. Ahrens, J. Glenn, J. Sandberg, and N. Tsoupas \\ Collider-Accelerator Department, Brookhaven National Laboratory, Upton, NY 11973
}

\begin{abstract}
Beam coupling phenomena have been observed in most fast kicker systems through out Brookhaven ColliderAccelerator complex. With ever-higher beam intensity, the signature of the beam becomes increasingly recognizable. The beam coupling at high intensity produced additional heat dissipation in high voltage modulator, thyratron grids, thyratron driver circuit sufficient to damage some components, and causes trigger instability. In this paper, we will present our observations, basic coupling mode analysis, relevance to the magnet structures, issues related to the existing high voltage modulators, and considerations of the future design of the fast kicker systems.
\end{abstract}

\section{MAGNET STRUCTURE}

The AGS G10 extraction fast kicker magnet has four identical sections. Its magnet window has a C-shaped small aperture. During beam injection and acceleration, the beam travels outside of the magnet gap. Only during fast extraction, the beam will be bumped into the magnet gap. The magnet conductor is centre-feed from the middle of the longitudinal dimension.

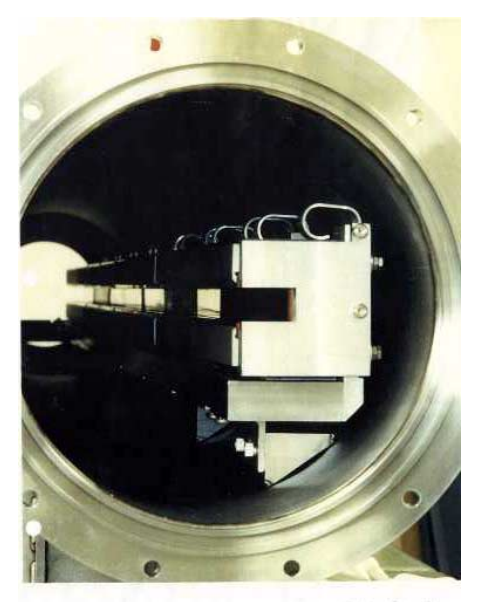

Picture 1. AGS G10 Extraction Fast Kicker Magnet and Vacuum Chamber

The AGS A5 injection kicker magnet and Booster F3 extraction kicker magnet are multi-section window frame magnets. The circulating beam will travel through the magnet aperture during injection, accumulation, acceleration and extraction.

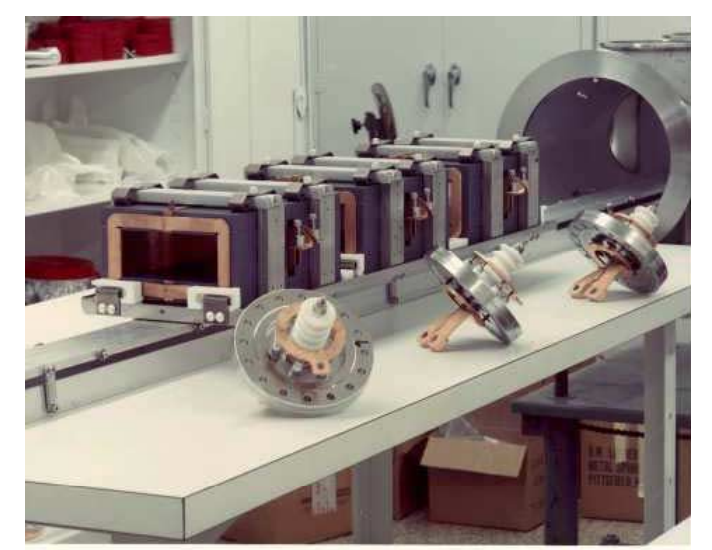

Picture 2. The AGS A5 Injection Fast Kicker Magnet

\section{OBSERVATION}

The beam coupling through kicker magnet has raised our attention in the recent years. It was treated as noise while intensity was low. During the 1999 high intensity run for g-2 experiment, we observed the beam intensity related kicker timing shift and investigated further to identify image current as the primary cause.

\subsection{Beam Coupling Induced Timing Shift}

The timing shift (trigger to current rise) at AGS G10 extraction fast kicker was measured under different conditions.

1. Accelerator running with no beam;

2. Running with multiple bunches, and extracting beam bunch by bunch in multiple transfers.

When the G10 kicker is pulsing with no beam, the timing is very solid (+/- $2 \mathrm{nS}$ jitter) and no erratic behaviour was observed. During extraction, the beam bunches are brought into $\mathrm{C}$-shaped kicker gap each time before a single bunch is to be transferred from the AGS ring.

The Figure 1 is a sequential measurement of twelve consecutive kicker pulses during high intensity single bunches multiple extractions. The trailing edge positions, as seeing by the end of waveform capture of each fragment relative to the delayed RF trigger, demonstrate systematic trigger timing shift over the twelve kicks.

"Work performed under the auspices of the U.S. Dept. of Energy. 




Figure 1. The AGS G10 pulse-timing shift during twelve extractions

\subsection{Beam Position Dependency}

The waveforms below show the beam current coupling measured at G10 kicker.

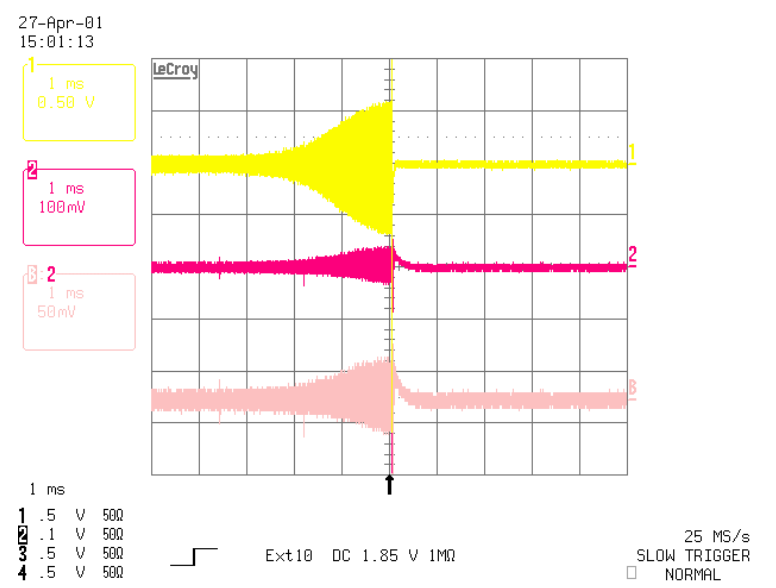

Figure 2. The beam coupling at AGS G10 Extraction Fast Kicker before and after the last bunch was extracted.

In Figure 2, the beam current coupling of the last beam bunch, extracted at the middle of the trace, is shown. The first trace was taken from trigger output monitor point. The second trace was the pulse current of the kicker magnet. The third one is enlargement of second trace. After the last beam bunch was extracted from the ring, the signal become clean. The half-sine bump that pushes the beam into the kicker gap rise in $3.5 \mathrm{mS}$. Figure 2 shows the position dependency of the beam coupling to the magnet conductor.

The Booster F3 is a full turn extraction kicker. It extracts all beam bunches out of Booster at a single pulse. Figure 3 is the beam coupling measured at the Booster F3 kicker. It shows a single beam bunch circulating in the Booster ring. This waveform was taken before Booster beam extraction. In this period, the beam was moved very

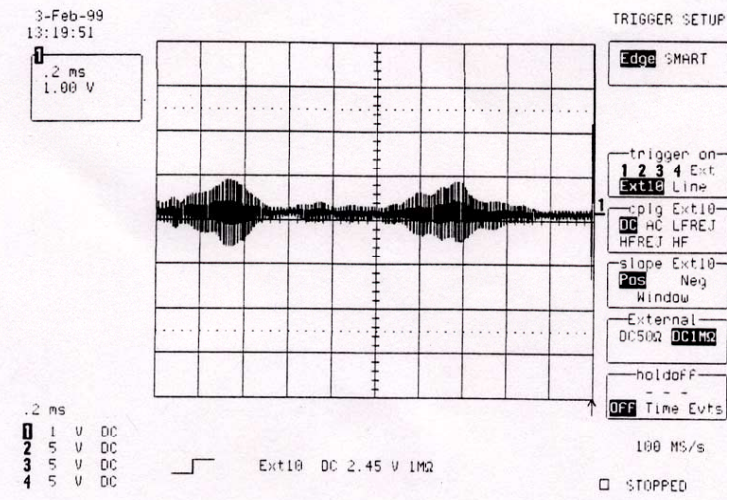

Figure 3. The circulating beam coupling at Booster F3 kicker.

close to the outer conductor of the magnet. Although this causes high beam current coupling, the extraction condition remain unchanged pulse to pulse provided the beam intensity remains stable. Therefore, the kicker has no apparent jitters. But the beam power coupled to the kicker thyratron driving circuit might damage the components.

\subsection{Beam Intensity Dependency}

Figure 4 is a sequential measurement of beam current coupling of AGS G10 during twelve consecutive bunch extractions at AGS. Before the first bunch was extracted, there were twelve bunches inside the machine. After the twelfth bunch was extracted, only residual beam remain in the ring. The correlation of the total beam intensity and the coupling is clearly indicated.

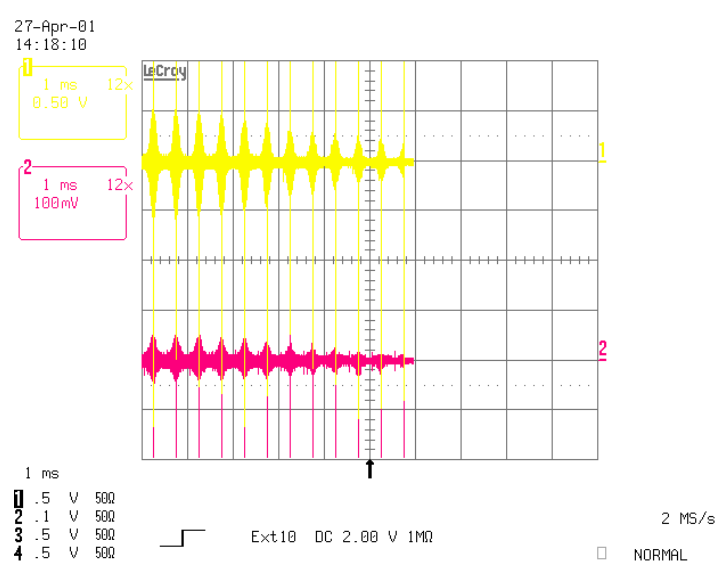

Figure 4. The beam coupling at AGS G10 during 12 consecutive single bunch multiple extraction.

Six beam transfers from Booster complete the AGS injection process. The total amount of beam inside the AGS ring increases as more and more beam being transferred into it. Hence the amount of coupled beam current at A5 Injection Kicker varies during each transfer. The Figure 5 is a sequential measurement of beam current coupling at AGS A5 injection kicker during six transfers 
before injection pulse. The waveform of channel 2, tailbiting current transformer signal, matches beam accumulation pattern clearly.

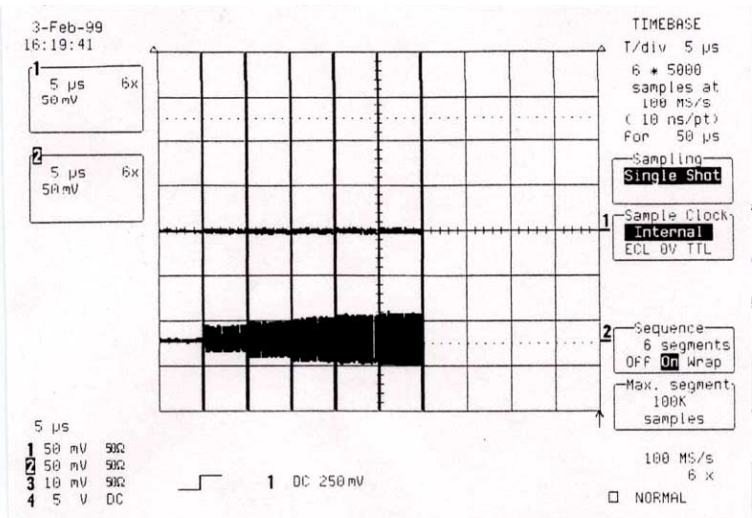

Figure 5. The beam coupling at AGS A5 before each of the six consecutive injections into AGS

The beam image current coupling has also been observed at RHIC beam abort kicker systems during heavy ion commissioning.

\section{DIFFERENTIAL IMAGE CURRENT COUPLING}

Multiple coupling modes, electrostatic and magnetic, are considered for beam coupled into ferrite magnet.

The beam inside the magnet window gap will use the magnet conductors, instead of vacuum chamber wall, as its image current return path. Since, the magnet conductor is not a closed loop, the beam image current must flow through the external circuit to complete its current loop. The external components in parallel to the conductor gap therefore serve as the current path. In the electrostatic coupling [1], the beam travelling off-centre of the magnet gap induces differential image current flow in opposite directions on the either side of the magnet conductors. The amplitude of the image current [1] is given by $I_{W}=-\frac{1}{2} \frac{\Delta}{b} I$, where $\mathrm{I}$ is the beam current, $\mathrm{b}$ is the distance from the gap centre to the side conductor, and $\Delta$ is the distance between the centre of the beam to the centre of the magnet gap.

When the beam is very close to one side of the magnet conductor, the magnitude of the image current will approach to one half of the beam current. However, the impedance associated with the external circuit is not necessarily resistive, it might be reactive impedance, such as the thyratron internal capacitance, PFN capacitance, etc. For instance, the G10 components in parallel to the magnet conductor gap are a thyratron in serial with a 6.5nF Capacitor, as shown in Figure 6a. At opening state, the thyratron is capacitive. Its gap capacitance and grid capacitance are in the tens of Pico-farad range. G10 magnet and external loop inductance is about $2.0 \mathrm{uH}$. The measured oscillation frequency of the coupled image current at the AGS G10 kicker, as shown in the Figure 6b, has a good agreement of this scenario. Here, the peak amplitude of the coupled image current is about $6.74 \mathrm{Amp}$ at 12-bunch, $52 \times 10^{12}$ total proton beam intensity. This current signal pumping through thyratron induces a voltage about thousand volts, and is strong enough to heat up the grid and break down the ionised gas.



Figure 6a. Simplified G10 Main Diagram

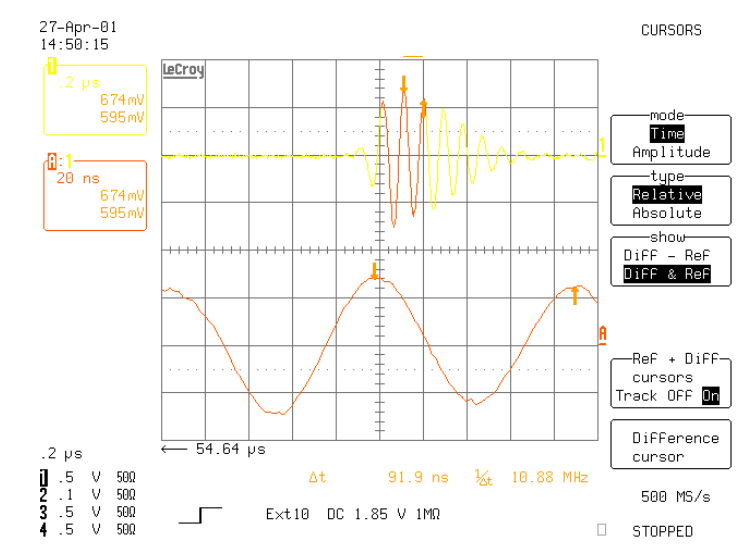

Figure 6b. Oscillation Frequency of the coupled beam current at AGS G10 kicker

The bias voltages at G10 thyratron were lowered to compensate this effect. The kicker design parameter given at early 90 s ignored beam-induced effects. The magnet gap of G10 kicker is very small, only $22 \mathrm{~mm}$ vertical by $32 \mathrm{~mm}$ horizontal to allow air operation voltage of less than $35 \mathrm{kV}$. In addition, the external packaging was made to minimize the stray inductance with a very tight metal enclosure for pulse current return, which has no enough space to add high voltage parallel resistor to bypass the image current. The high radiation level resulting from high beam loss at AGS G10 magnet combining with the beam image current problem has made some damages on the high voltage modulator components. However, the access to the high voltage modulators is increasingly difficult due to the high level radiation from excessive beam loss at the AGS G10 area. Further study will be conducted for better understanding.

\section{REFERENCES}

[1] F.J. Sacherer, "Transverse Bunched-Beam Instabilities," CERN/PS/BR 76-21. 